\title{
Judging a virus by its cover
}

\author{
Eva Szomolanyi-Tsuda and Raymond M. Welsh
}

Department of Pathology, University of Massachusetts Medical School, Worcester, Massachusetts, USA.

\begin{abstract}
The production of protective neutralizing antibodies occurs quickly in some viral infections but very slowly in others. In a new study, surface glycoproteins (the targets of neutralization) of 2 different viruses were genetically switched. Analysis of the neutralizing antibody response to each of the 2 parent and recombinant viruses in infected mice revealed that the speed of neutralizing antibody induction was intrinsically dependent on the surface glycoprotein and not the rest of the virus (see the related article beginning on page 988 ).
\end{abstract}

We are taught not to judge a book by its cover, but the "cover" of a virion may be all that concerns the immune system when it comes to its speed in generating neutralizing antibodies. Neutralizing antibodies are a major component of the immune defense mechanism against viral infections. These antibodies bind to accessible surface determinants on virions, including attachment proteins, and render viruses noninfectious. Mechanisms of neutralization include antibody interference with the virus binding to host cell surface receptors and the blocking of viral fusion with the host cell membrane, thereby preventing the entry of infectious virions (1). Antiviral neutralizing antibodies protect the host from reinfection, keep low-grade, persistent infections from recrudescing, and prevent infection when administered or elicited prophylactically. For this reason, the efficient induction of high titers of neutralizing antibodies is a major goal in vaccine design.

\section{Variations in the timing of neutralizing antibody induction}

Many viral infections induce neutralizing antibody responses rapidly. These responses can be detected as early as days 3-7 of infection with rota and vesicular stomatitis viruses (VSVs) in mice, rabies and yellow fever viruses in humans, and influenza and polio viruses in both humans and mice (2-5). The generation of neutralizing

Nonstandard abbreviations used: AID, activationinduced deaminase; LCMV, lymphocytic choriomeningitis virus; rLCMV, recombinant LCMV; rLCMV/ VSV-GP, rLCMV expressing VSV-glycoprotein; rVSV, recombinant VSV; rVSV/LCMV-GP, rVSV expressing LCMV-glycoprotein; SIV, simian immunodeficiency virus; VSV, vesicular stomatitis virus.

Conflict of interest: The authors have declared that no conflict of interest exists.

Citation for this article: J. Clin. Invest. 114:895-897 (2004). doi:10.1172/JCI200423098. antibodies early during the course of infection enables them to participate in virus clearance; in some infections, such as VSV, neutralizing antibodies play major roles in the resolution of acute infection and recovery. In other viral infections there is a long delay between initial infection and the generation of high levels of neutralizing antibodies. Such delays may extend from one to several months and are often observed in infections with hepatitis $C$ virus, hepatitis $B$ virus, and HIV in humans, and with lymphocytic choriomeningitis virus (LCMV) in humans and mice (6-9). In this issue of the JCI, Pinschewer and colleagues pose the following question: What factor(s) determine the timing of the onset of efficient neutralizing antibody responses to viral infection (10)? The ability of a virus to induce neutralizing antibodies early in the course of infection could be due either to: (a) an inherent property of the viral protein target of neutralization antibodies; (b) the organization or topography of the virion antigen display, which may influence the triggering of the immunoglobulin receptor on $B$ cells and the subsequent activation of antibody-secreting $B$ cells $(11,12)$; or (c) the nature of the virus infection, including the ability of the virus to propagate in antigen-presenting cells, induce cytokines, and induce and exhaust $\mathrm{T}$ cell immune responses that may affect the generation of antibody responses.

\section{Swapping viral glycoproteins}

Pinschewer et al. (10) used a genetic approach to address the question of what makes some viruses proficient at the rapid induction of high titers of neutralizing antibodies. They made recombinant viruses of VSV (called recombinant VSV, or rVSV), a potent inducer of neutralizing antibodies, and also of LCMV (called recombinant LCMV, or rLCMV), an inefficient inducer of neutralizing antibodies, by swapping their surface glycoproteins, which are targets of antibody-mediated neutralization. This resulted in rLCMV expressing VSV-glycoprotein (rLCMV/ VSV-GP) and rVSV expressing LCMV-glycoprotein (rVSV/LCMV-GP). The authors then compared the neutralizing antibody responses to each of the 2 parent and recombinant viruses in infected mice. The results suggest a simple and at first surprising answer (Figure 1). The responses to the recombinant viruses were determined exclusively by the surface glycoprotein and not by the rest of the virus. rLCMV/VSVGP induced rapid and efficient neutralizing antibody responses (Figure 1D), similar to the responses induced by the parental VSV strain (Figure 1A); mice infected with rVSV/LCMV-GP produced few detectable neutralizing antibodies during the 30-day observation period (Figure 1C), similarly to mice infected with LCMV (Figure 1B). Most other parameters of infection with parental LCMV and rVSV/LCMV-GP were similar or the same, including the induction of $\mathrm{T}$ cell responses, even though the quantity of viral antigen and cellular tropism of the recombinants may have been influenced by the surface glycoprotein.

\section{Are differences in timing due to germ-line immunoglobulin sequences?}

How then could the intrinsic properties of the viral surface glycoprotein account for the timing of neutralizing antibody responses? In some cases, highly glycosylated proteins, such as the HIV or simian immunodeficiency virus (SIV) envelope glycoproteins, can only induce low titers of neutralizing antibodies. Removal of glycosylation sites of the SIV glycoprotein allows the induction of antibodies with increased neutralizing activity (13). The high levels of glycosylation, however, may interfere with the ability of the virus to induce high titers of neutralizing antibodies, but do not affect the timing of neutralizing antibody production.

The answer to the question posed by Pinschewer and colleagues may have more to do with how the antibody response 
A

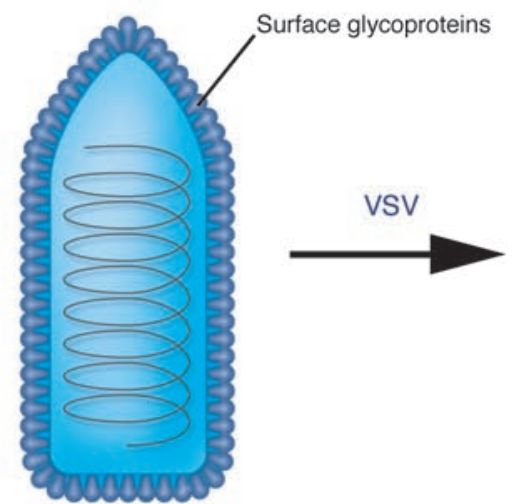

B

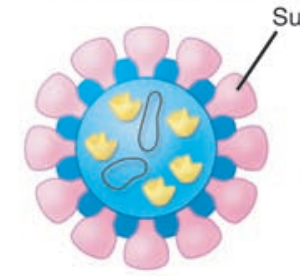

artaros
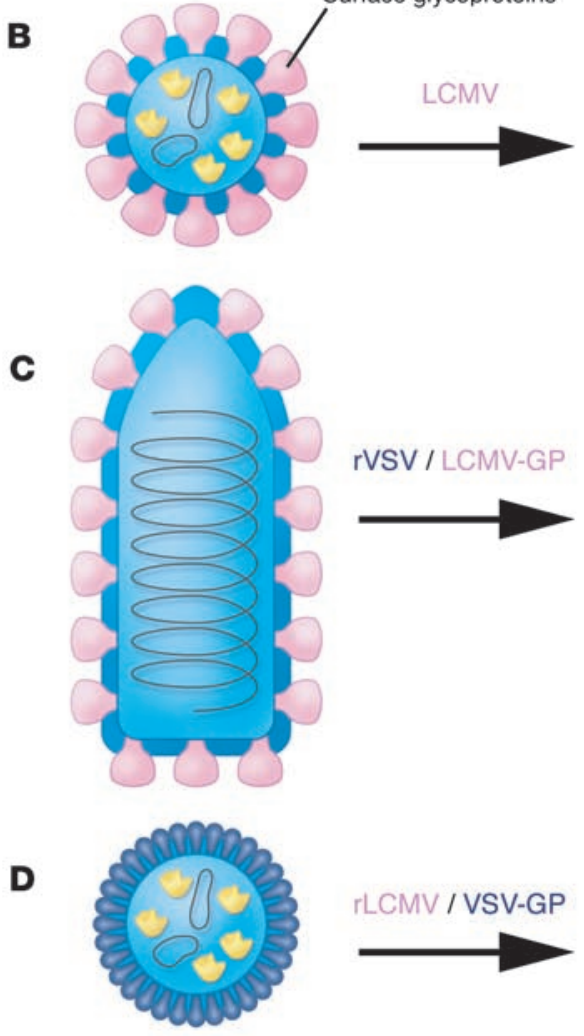

by Bruce Worden, J. Clin. Invest.

ILCMV / VSV-GP

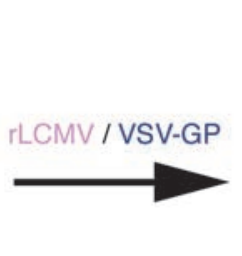

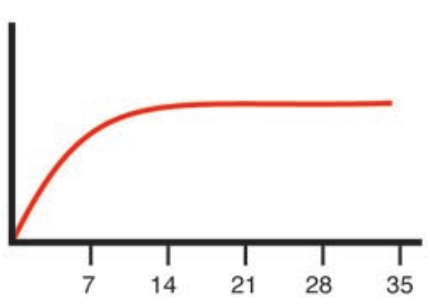
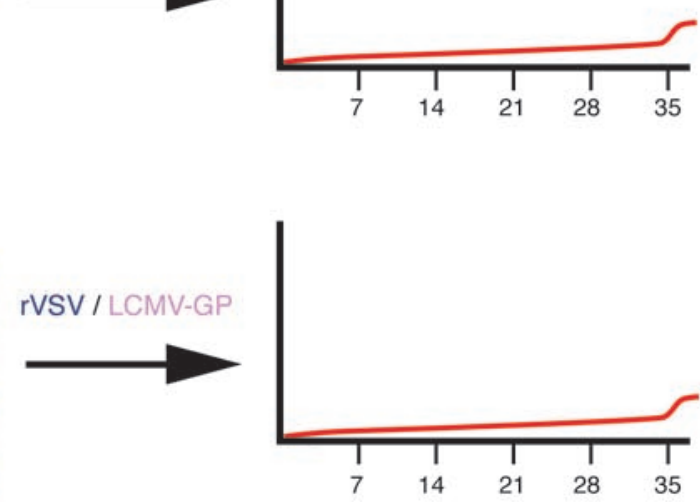

I

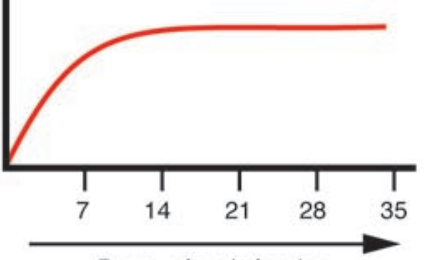

Days after infection

\section{Figure 1}

Kinetics of neutralizing antibody responses induced in mice following infection with (A) VSV, a bullet-shaped rhabdovirus containing one RNA species and (B) LCMV, an arenavirus containing 2 virion RNAs and some ribosomes. By reverse genetic techniques, the virion surface glycoproteins were swapped between the two viruses (C and $\mathbf{D}$ ), and the rapidity in generating neutralizing antibodies was found to correlate with the surface glycoprotein expressed on the recombinant virus (10).

tion, although somatic hypermutation does occur after days 6-8 in the germinal centers $(15,16)$. The high, germline-encoded affinities enable VSV to elicit protective neutralizing IgM antibodies on days 3-4. These IgM antibodies are generated in a helper $\mathrm{T}$ cell-independent fashion (12). Hence, this capacity to secrete Ig that is reactive with a virus at high affinity at the start of the $\mathrm{B}$ cell response may explain the rapid neutralizing antibody induction in response to VSV glycoproteins $(10,12)$.

A remaining question is whether the induction of a high-affinity, germlineencoded, protective antibody response during early infection is a phenomenon unique to VSV infection in the mouse. Does this response occur during infection of other host species with viruses other than LCMV and VSV, or does the study of Pinschewer et al. (10) merely illustrate a curious exception? Some of the other viruses known to induce early neutralizing antibody responses, such as influenza and polio, induce early, protective IgM responses. These early IgM responses to influenza virus are $\mathrm{T}$ cell-independent (17), and influenza virus induces protective antibody responses in mice deficient in activation-induced deaminase (AID), an enzyme essential for both isotype switching and somatic hypermutation (18). In the AID-deficient mice, the unmutated IgM must have met the affinity requirements necessary for virus neutralization and host protection. Influenza virus-specific antibody responses, like those to VSV, eventually undergo somatic hypermutation (19), but this process is not required for their protective or neutralizing capacity. Detailed studies on antiviral neutralizing antibody affinities at different stages of infection have not been done in other viral systems.

In the event that a viral infection induces germline-encoded, high-affinity antibodies specific to the viral surface proteins, there 
would be no need for $\mathrm{T}$ cell help, formation of germinal centers, and the time-consuming process of somatic hypermutation and selection before the neutralizing antibodies are produced. In contrast, the late-forming neutralizing antibody responses to viruses such as HIV, hepatitis B virus, hepatitis $\mathrm{C}$ virus, and LCMV, may require affinity maturation in order to reach neutralization potential. Additional challenges in containing these infections may also be envisioned as a result of the ability of all of these viruses to either replicate within or induce clonal exhaustion of T cells, which are needed for the affinity maturation process described above.

There may not be a quick and simple solution for rapidly eliciting neutralizing antibody responses to certain viruses if the results obtained by Pinschewer et al. (10) with the recombinant LCMV and VSV with swapped surface glycoproteins reflect a general rule. The initial affinity of antibodies to a given viral protein cannot be altered by placing the epitope into a new, highly immunogenic carrier. Fortunately, most immunization strategies allow sufficient time for neutralizing antibodies to develop prior to exposure to the infectious virus, independent of whether the viral antigens are characterized as potent or inefficient inducers of neutralizing antibodies.

\section{Acknowledgments}

We thank Keith Daniels for help with the figure. The authors were supported by grants CA66644 (EST), AI17672, AR35506, and CA34461 from the NIH.

Address correspondence to: Raymond M. Welsh, Department of Pathology, University of Massachusetts Medical School, Worcester, Massachusetts 01655, USA. Phone: (508) 856-5819; Fax: (508) 856-0019; E-mail: raymond.welsh@umassmed.edu.

1. Dimmock, N.J. 1993. Neutralization of animal viruses. Curr. Top. Microbiol. Immunol. 183:1-149.

2. Franco, M.A., and Greenberg, H.B. 1999. Immunity to rotavirus infection in mice. J. Infect. Dis. 179(Suppl. 3):S466-S469.

3. Ochsenbein, A.F., et al. 2000. Correlation of T cell independence of antibody responses with antigen dose reaching secondary lymphoid organs: implications for splenectomized patients and vaccine design. J. Immunol. 164:6296-6302.

4. Tomori, O. 1999. Impact of yellow fever on the developing world. Adv. Virus Res. 53:5-34

5. Gerhard, W., et al. 1997. Role of B cell response in recovery of mice from primary influenza virus infection. Immunol. Rev. 159:95-103.

6. Ferrantelli, F., and Ruprecht, R.M. 2002. Neutralizing antibodies against HIV - back in the major leagues? Curr. Opin. Immunol. 14:495-502.

7. Farci, P., et al. 1994. Prevention of hepatitis $C$ virus infection in chimpanzees after antibody-mediated in vitro neutralization. Proc. Natl. Acad. Sci. U. S. A. 91:7792-7796.

8. Bachmann, M.F., and Zinkernagel, R.M. 1997. Neutralizing antiviral B cell responses. Annu. Rev. Immunol. 15:235-270.
9. Lehmann-Grube, F., et al. 1979. Serologic diagnosis of human infections with lymphocytic choriomeningitis virus: comparative evaluation of seven methods. J. Med. Virol. 4:125-136.

10. Pinschewer, D.D., et al. 2004. Kinetics of protective antibodies are determined by the viral surface antigen. J. Clin. Invest. 114:988-993. doi:10.1172/ JCI200422374.

11. Szomolanyi-Tsuda, E., et al. 1998. T-cell-independent immunoglobulin $G$ responses in vivo are elicited by live-virus infection but not by immunization with viral proteins or virus-like particles. J. Virol. 72:6665-6670.

12. Bachmann, M.F., et al. 1995. T helper cell-independent neutralizing $\mathrm{B}$ cell response against vesicular stomatitis virus: role of antigen patterns in B cell induction? Eur. J. Immunol. 25:3445-3451.

13. Reitter, J.N., et al. 1998. A role for carbohydrates in immune evasion in AIDS. Nat. Med. 4:679-684

14. Bachmann, M.F., et al. 1997. The role of antibody concentration and avidity in antiviral protection. Science. 276:2024-2027.

15. Kalinke, U., et al. 1996. The role of somatic mutation in the generation of the protective humoral immune response against vesicular stomatitis virus. Immunity. 5:639-652.

16. Roost, H.-P., et al. 1995. Early high-affinity neutralizing antiviral IgG responses without overall improvements of affinity. Proc. Natl. Acad. Sci.U.S. A. 92:1257-1261.

17. Mozdzanowska, K., et al. 2000. Th cell-deficient mice control influenza virus infection more effectively than Th- and B cell-deficient mice: evidence for a Thindependent contribution by B cells to virus clearance. J. Immunol. 164:2635-2643.

18. Harada, Y., et al. 2003. Unmutated immunoglobulin $\mathrm{M}$ can protect mice from death by influenza virus infection. J. Exp. Med. 197:1779-1785.

19. Clarke, S., et al. 1990. The BALB/c secondary response to the Sb site of influenza virus hemagglutinin. Nonrandom silent mutation and unequal numbers of $\mathrm{VH}$ and Vk mutations. J. Immunol. 145:2286-2296. 